\title{
Regional Mutational Signature Activities in Cancer Genomes
}

\author{
Caitlin Timmons $^{\mathrm{a}, \mathrm{d}}$, Quaid Morris $\mathrm{s}^{\mathrm{a}, \mathrm{b}, \mathbb{}, \text { and Caitlin F. Harrigan }}{ }^{\mathrm{b}, \mathrm{c}, \mathbb{}}$ \\ ${ }^{a}$ Computational \& Systems Biology, Sloan Kettering Institute, New York, USA \\ ${ }^{\mathrm{b}}$ Vector Institute for Artificial Intelligence, Toronto, Canada \\ ${ }^{c}$ Department of Computer Science, University of Toronto, Toronto, Canada \\ ${ }^{\mathrm{d}}$ Department of Biological Sciences, Smith College, Northampton, USA
}

\begin{abstract}
Cancer genomes harbor a catalog of somatic mutations. The type and genomic context of these mutations depend on their causes, and allow their attribution to particular mutational signatures. Previous work has shown that mutational signature activities change over the course of tumor development, but investigations of genomic region variability in mutational signatures have been limited. Here, we expand upon this work by constructing regional profiles of mutational signature activities over 2,203 whole genomes across 25 tumor types, using data aggregated by the Pan-Cancer Analysis of Whole Genomes (PCAWG) consortium. We find that 426 genomes from 20 tumor types display at least one change in mutational signature activities (changepoint), and $\mathbf{1 7 4}$ genomes contain a recurrent changepoint shared by three or more genomes of the same tumor type. Twenty-three recurrent changepoint locations are shared by multiple tumor types. Within these regions, the particular signature changes are often consistent across samples of the same type, and some, but not all are characterized by signatures associated with subclonal expansion. The changepoints we found cannot strictly be explained by gene density, mutation density, or cell-of-origin chromatin state. We hypothesize that they reflect a confluence of factors including evolutionary timing of mutational processes, regional differences in somatic mutation rate, large-scale changes in chromatin state that may be tissue type-specific, and changes in chromatin accessibility during subclonal expansion. These results provide insight into the regional effects of DNA damage and repair processes, and may help us localize genomic and epigenomic changes that occur during cancer development.
\end{abstract}

Mutational signatures | Cancer evolution | Whole genome sequencing Correspondence: morrisq@mskcc.org charrigan@cs.toronto.edu

\section{Introduction}

Cancer is a disease that develops over a lifetime through a series of somatic mutations. The vast majority of these mutations are "passenger mutations", which have little effect on fitness. Various mutagenic processes operate in a cancer throughout its development, which in turn determine the nature of the collection of somatic mutations that accumulate. Different mutagenic processes give rise to distinct mutational patterns, called mutational signatures (Alexandrov et al., 2020).

In nearly all cancers, multiple mutagenic processes contribute somatic mutations to individual cancer genomes. The relative contributions of these different processes can be quantified by algorithms that assign an activity (or exposure) to each detected mutational signature (Alexandrov et al., 2020). Recent work (Dentro et al., 2021, Gerstung et al., 2020, Rubanova et al., 2020) has revealed that i) these activities vary during cancer development (i.e., tumor progression) in a cancertype-specific way and ii) most mutational signatures are primarily active only in either early- or late-tumor progression (Dentro et al., 2021, Gerstung et al., 2020) and methods, like TrackSig, have been developed to reconstruct the evolutionary trajectories of mutational signature activities in individual samples (Rubanova et al., 2020, Harrigan et al., 2020). Taken together, this indicates that mutational signature analysis is a compelling tool in our search for understanding of the processes shaping tumor formation and development.

In addition to evolutionary timing of signature activity, somatic mutation rate and mutational signatures have been found to be influenced by genomic and epigenomic factors that exert their effects on the scale of several nucleotides up to a megabase. These factors include sequence context, nucleosome positioning, chromatin state, and replication timing (e.g. Gonzalez-Perez et al. (2019), Haradhvala et al. (2016), Hodgkinson et al. (2012), Lawrence et al. (2013), Polak et al. (2014, 2015), Schuster-Böckler and Lehner (2012), Seplyarskiy and Sunyaev (2021), Supek and Lehner (2019), Vöhringer et al. (2021), Yaacov et al. (2021)). For example, late-replicating and heterochromatic regions accumulate more mutations than early-replicating and euchromatic regions (Supek and Lehner, 2019, 2015, Zheng et al., 2014). This results in tumors accumulating mutations in patterns that can be attributed to chromatin state in the cell of origin. In mismatch repair (MMR)-proficient tumors, the rate of $\mathrm{CpG}>\mathrm{TpG}$ mutations may correlate with replication timing since MMR corrects mispairings due to $5 \mathrm{mC}$ deamination more efficiently in early-replicating areas (Gonzalez-Perez et al., 2019, Supek and Lehner, 2019).

These genomic features vary on scales of $1 \mathrm{Mb}$ or less, so analyses of their effects on mutational signature activity are normally confined to particular loci. To our knowledge, the relationship between chromosomal location-over the scale of an entire genome-and mutational signature activity has not been thoroughly investigated. If there exists common evolutionary events that dictate the action of mutational processes in cancers of the same type, we can expect to observe consistent changes to mutational signature activity across genomes.

To examine the occurrence of mutational signature activity changes across the genome, we analyzed somatic mutations of 2,203 whole genomes across 25 tumor types from 
the Pan-Cancer Analysis of Whole Genomes (PCAWG) consortium (Campbell et al., 2020). Using an extension of the TrackSig method (Rubanova et al., 2020), we identified genomic regions that recurrently exhibit mutational signature changes. These regions frequently showed activity changes in signatures known to be associated with evolutionary timing. Some of these regions were shared among several tissue types, while others appeared to be exclusive to specific tissue types.

\section{Methods}

We created an extension of the TrackSig algorithm (Rubanova et al., 2020, Harrigan et al., 2020) to variations in mutation signature activity across the genome. TrackSig is designed to detect evolutionary changes in mutational signature activity by segmenting a ranked list of mutations, sorted by inferred cancer cell frequency into subsequences, i.e. segments, of at least 100 mutations with constant mutation signature activity. We extended TrackSig so that the mutations could be sorted instead via chromosomal location and so that it could be run separately across different chromosomes in the same genome. Thus our new method GenomeTrackSig uses the PELT segmentation algorithm (Killick et al., 2012) to identify regions of the genome where signature activities change (changepoints) while using the EM algorithm (Moon, 1996) to fit a multinomial mixture model to estimate signature activities within each segment defined by changepoints. Previous work by Alexandrov et al. (2020) characterized canonical signatures active within the PCAWG data. These serve as the set of reference signatures for our analyses. We fit activity estimates to all signatures which have been previously determined (Alexandrov et al., 2020) to be active in a given tumor type.

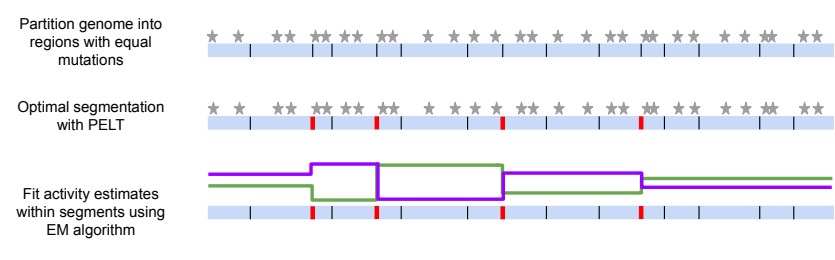

Fig. 1. Overview of GenomeTrackSig algorithm for profiling mutational signature activities across the genome. Stars indicate mutations (top); red bars indicate changepoints (middle); green and purple lines indicate estimated mutation signature activities; $y$-axis indicates exposure level (bottom). GenomeTrackSig requires at least 100 mutations per segment, fewer mutations are shown here for illustrative purposes.

For efficiency, we partition the set of mutations in a genome into chromosomal regions with equal numbers of mutations, although the number of bases spanned by a region will vary depending on mutation density. We require each segment to include at least one region, so segments have at least one region's worth of mutations. GenomeTrackSig includes flexible options for analyzing samples, either by partitioning and estimating signature activities across an entire genome or on each individual chromosome. The genome-wide approach can capture signature activities that span multiple chromosomes thereby potentially reducing noise in activity estimates at chromosomal ends. On the other hand, the chromosomewise approach may be more sensitive to activity changes within a chromosome and substantially reduces runtime on samples with many mutations. In general, the chromosomewise approach is preferred, but we use the genome-wide strategy for samples (and cancers) where chromosomes have less than 100 mutations, as we use this as a minimum segment size to ensure accurate activity estimates.

\section{Results}

A. Mutational signature activity is not constant across the genome. We constructed genome-wide signature activity profiles for 2,203 tumors across 25 tumor types (Biliary-AdenoCA, Bladder-TCC, Bone-Osteosarc, BreastAdenoCA, Cervix, CNS-GBM, Colorect-AdenoCA, EsoAdenoCA, Head-SCC, Kidney-ChRCC, Kidney-RCC, LiverHCC, Lung-AdenoCA, Lung-SCC, Lymph-BNHL, LymphCLL, Melanoma, Myeloid-MPN, Ovary-AdenoCA, PancAdenoCA, Panc-Endocrine, Prost-AdenoCA, StomachAdenoCA, Thy-AdenoCA, Uterus-AdenoCA). We found no genomic changepoints in five types of tumor: LiverHCC $(n=326)$, Ovary-AdenoCA $(n=113)$, Biliary-AdenoCA $(n=29)$, Panc-Endocrine $(n=76)$, and Myeloid-MPN $(n=31)$. A lack of change in signature activity could reflect an even mutational composition across the genome, insufficient number of mutations to characterize a change in signature activity, a lack of large-scale variation in chromatin accessibility, or a lack of substantial changes in mutational composition over tumor development. In the remaining 20 tumor types, at least one sample exhibited changes in signature activities across the genome, indicating that large-scale shifts in signature activities depending on chromosomal location are a common but not ubiquitous phenomenon.

\section{B. Some changepoints are shared across tissue type} .We find that changepoint regions are often shared-both across multiple cancers from the same tissue and across multiple tissues. To determine which changepoints within samples from the same tumor type overlap, we fit a kernel density estimate across the vector of genomic locations covered by each changepoint and constructed a possible range for that changepoint location, which contains the changepoint locus identified by GenomeTrackSig, +/- one standard deviation of the density function. We then overlaid all changepoint ranges from that tumor type and counted how many changepoints fell within a sliding window across the genome. Regions with changepoints supported by less than three samples were excluded from consideration. We then deemed a 'recurrent changepoint region' for each tumor type as the center of the region where many samples shared an overlapping changepoint; i.e. the window within a region in which the maximum number of changepoints fell. Of the 20 tumor types that show any changes in signature activity across their genomes, 12 contain recurrent changepoint regions (Fig. 2). Interestingly, the changes in signature activity at these regions are often highly similar across samples, both in direction and magnitude. For example, all 55 melanoma 
bioRxiv preprint doi: https://doi.org/10.1101/2022.01.23.477261; this version posted February 27, 2022. The copyright holder for this preprint (which was not certified by peer review) is the author/funder, who has granted bioRxiv a license to display the preprint in perpetuity. It is made available under aCC-BY-NC 4.0 International license.

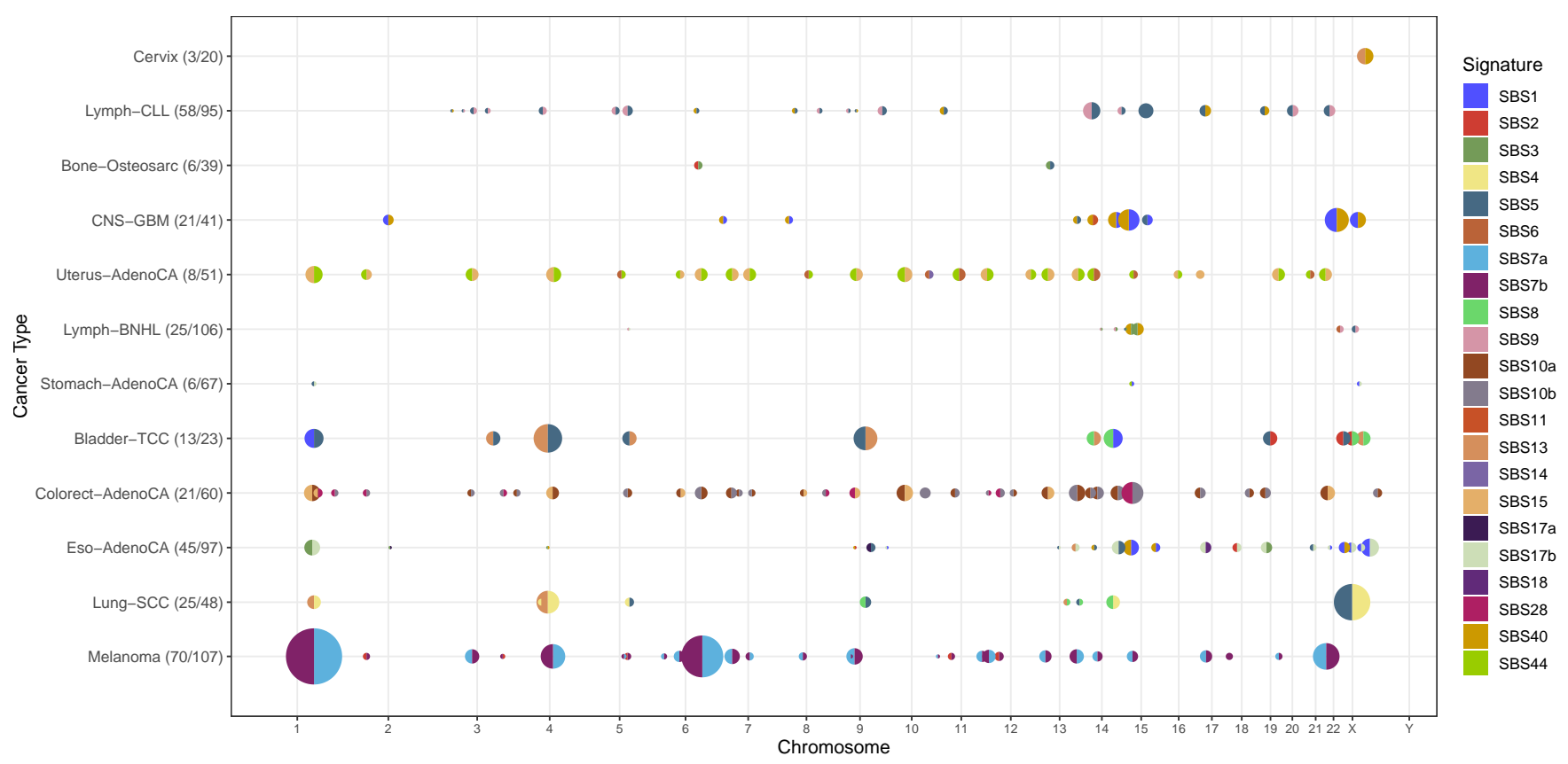

Fig. 2. Recurrent changepoint regions across 12 tumor types. We observe changepoints in 426/2,203 samples. One hundred seventy-four genomic regions are covered by a changepoint found in more than two samples for a given tumor type. Each of these recurrent changepoint regions are indicated by a point, where COSMIC V3 signatures (Alexandrov et al., 2020, Tate et al., 2019) are indicated by color. Changepoints are summarized by the signature whose activity decreases the most on average (left half) and the signature whose activity increases the most on average (right half). Proportion of samples exhibiting a changepoint is encoded by size, and the number of samples affected is indicated in the tumor type label $(\mathrm{n} / \mathrm{N})$. Changepoints that appear in fewer than three samples are omitted.

\begin{tabular}{|c|c|c|c|c|c|c|c|c|}
\hline Tumor Type & $\begin{array}{l}\text { Samples with at least one } \\
\text { changepoint } / \mathrm{N} \text { total samples }\end{array}$ & $\begin{array}{c}\text { Mean } \\
\text { \# changepoints } \\
\text { per sample }\end{array}$ & $\begin{array}{c}\text { Median } \\
\text { \# changepoints } \\
\text { per sample }\end{array}$ & $\begin{array}{c}\text { Mean } \\
\text { changepoint } \\
\text { magnitude }\end{array}$ & $\begin{array}{c}\text { Median } \\
\text { changepoint } \\
\text { magnitude }\end{array}$ & $\begin{array}{c}\text { Top } 3 \text { signatures } \\
\text { with greatest changes }\end{array}$ & Clonal signatures & Subclonal signatures \\
\hline Melanoma & $74 / 107$ & 11 & 4 & 0.15 & 0.13 & $7 \mathrm{~b}, 7 \mathrm{a}, 2$ & $7 \mathrm{a}, 7 \mathrm{~b}$ & 5 \\
\hline Lung-SCC & $35 / 48$ & 9 & 4 & 0.05 & 0.04 & $4,13,8$ & 4 & $5,2,13$ \\
\hline Eso-AdenoCA & $50 / 97$ & 5 & 4 & 0.09 & 0.07 & $17 \mathrm{~b}, 40,1$ & 17 & 5,40 \\
\hline Lung-AdenoCA & $5 / 33$ & 2 & 2 & 0.16 & 0.11 & $5,13,2$ & 4 & 2,13 \\
\hline Colorect-AdenoCA & $21 / 60$ & 27 & 5 & 0.04 & 0.03 & $10 \mathrm{~b}, 15,10 \mathrm{a}$ & 1,44 & 18,40 \\
\hline Bladder-TCC & $16 / 23$ & 6 & 3 & 0.06 & 0.05 & $13,8,5$ & 2,13 & 5 \\
\hline Stomach-AdenoCA & $8 / 67$ & 3 & 2 & 0.13 & 0.11 & $17 b, 40,15$ & 1 & 18 \\
\hline Head-SCC & $1 / 54$ & 2 & 2 & 0.15 & 0.15 & $40,4,5$ & 5 & 2,13 \\
\hline Lymph-BNHL & $29 / 106$ & 3 & 2 & 0.2 & 0.15 & $3,9,6$ & 9 & $17 \mathrm{a}, 17 \mathrm{~b}, 40$ \\
\hline Uterus-AdenoCA & $9 / 51$ & 41 & 48 & 0.06 & 0.05 & $15,44,6$ & 1 & $2,13,40,44$ \\
\hline CNS-GBM & $24 / 41$ & 6 & 4 & 0.05 & 0.03 & $40,5,1$ & 1 & 40 \\
\hline Kidney-RCC & $8 / 144$ & 1 & 1 & 0.08 & 0.02 & $5,40,13$ & 40 & 1 \\
\hline Breast-AdenoCA & $18 / 193$ & 3 & 2.5 & 0.25 & 0.2 & $2,5,13$ & 3,5 & $2,3,13,18$ \\
\hline Panc-AdenoCA & $12 / 238$ & 2 & 2 & 0.38 & 0.34 & $2,13,1$ & 1,5 & $2,3,13,17 \mathrm{a}, 17 \mathrm{~b}, 18,40$ \\
\hline Bone-Osteosarc & $13 / 39$ & 3 & 2 & 0.31 & 0.35 & $2,5,3$ & 2,13 & 40 \\
\hline Prost-AdenoCA & $11 / 145$ & 2 & 2 & 0.17 & 0.16 & $13,5,40$ & 5 & 40 \\
\hline Lymph-CLL & $66 / 95$ & 3 & 3 & 0.22 & 0.19 & $5,40,9$ & 9 & 5 \\
\hline Kidney-ChRCC & $5 / 38$ & 4 & 4 & 0.24 & 0.22 & $2,5,40$ & 5 & $1,2,13,40$ \\
\hline Cervix & $13 / 20$ & 2 & 2 & 0.08 & 0.07 & $40,13,5$ & $1,2,13,5$ & 40 \\
\hline Thy-AdenoCA & $8 / 29$ & 2 & 1 & 0.2 & 0.15 & $5,40,1$ & 5 & 2,13 \\
\hline
\end{tabular}

Table 1. Overview of changepoints discovered across 20 cancer types. Magnitude of each changepoint is measured as the cosine distance of the signature activity vector on either side of the changepoint. For each cancer type containing changepoints, the three signatures with the greatest absolute value of activity changes across all changepoints in that cancer are listed. Signatures are indicated as "clonal" or "subclonal" respectively based on where their activity is highest, as described by Dentro et al. (2021).

samples that have a changepoint overlapping with the chromosome 1:47,000,000-1:51,000,000 region show a decrease in SBS7b activity and increase in SBS7a activity in the direction of the numbering (Fig. 3). In Uterus-AdenoCA samples, the signatures which changed most at every recurrent changepoint are associated with defective DNA mismatch repair, most consistently SBS15 and SBS44 (Fig. 2). In other cancers, the most affected signatures are highly variable from changepoint-to-changepoint: seven different signatures are represented among the most substantial activity changes at recurrent changepoints in Eso-AdenoCA samples, including SBS1, SBS2, SBS3, SBS5, SBS17a, SBS17b, and SBS40 (Fig. 2). We also find that 23 recurrent changepoints are shared by multiple tumor types. The most common recurrent changepoint region is 1:47,000,000-1:51,000,000, which appears in Melanoma, Uterus-AdenoCA, Lung-SCC, Bladder-TCC, and Stomach-AdenoCA (Fig. 2). Other recurrent changepoint regions shared by three tumor types 
include coordinates 1:190,000,000-1:194,000,000 (ColorectAdenoCA, Melanoma, Uterus-AdenoCA), 3:194,000,0003:198,000,000 (Bladder-TCC, Eso-AdenoCA, Lung-SCC), 5:22,000,000-5:26,000,000 (Colorect-AdenoCA, LymphCLL, Melanoma), and 13:94,000,000-13:98,000,000 (Bladder-TCC, Lung-SCC, and Uterus-AdenoCA) (Fig. 2). Despite the consistency of changepoint locations across multiple samples and tissues, it is unlikely that all these changepoints result from mutations in specific genes near the changepoint region. For one, changepoint regions span multiple megabases, so it is difficult to attribute the changepoint to mutations in any one particular gene. That said, some changepoint regions contain genes that may play a role in tumorigenesis such as Lymph-CLL changepoint regions encompassing CBL proto-oncogene B and NRP2 (Goel et al., 2012, Liyasova et al., 2015), and a changepoint region shared by Lung-SCC and Bladder-TCC which encompasses the DDX17 gene (Wu, 2020). However, we were unable to detect any clear pattern of gene function at changepoint regions.

\section{Many changepoints are characterized by signa- tures associated with subclonal expansion. In certain} cancers, we find that recurrent changepoints show a shift in activity from signatures associated with early cancer development to signatures associated with subclonal expansion. For instance, at 13/21 recurrent changepoints in Lymph-CLL samples, the most dramatic signature activity changes were increases in SBS9 and decreases in SBS5, or vice versa. We validated that these changepoints indeed reflect a shift between early and late signatures by constructing evolutionary trajectories for 90 Lymph-CLL samples using TrackSig (Rubanova et al., 2020). Figure 4 shows the distribution of activity changes from early to late development across all samples, in which we observe a definitive decrease in SBS9, and increase in SBS5 activity. In other cancers, signatures which account for the most dramatic activity changes at recurrent changepoint regions do not show a clear timing association. For example, SBS7a and SBS7b change most dramatically across the genome in melanoma samples (Fig. 2), yet show a weak association with evolutionary timing (from early-occurring to late-occuring mutations, SBS7a activity decreases by $8.7 \%+/-12.2 \%$ on average and SBS7b activity increases by $1.7 \%+/-9.1 \%$ on average).

Chronic lymphocytic leukemias (Lymph-CLL) tend to exhibit high SBS9 activity early in development, and show decreasing SBS9 and increasing SBS5 and SBS40 activities as subclones form (Fig. 4; Dentro et al. (2021)). SBS9 activity is attributed to mutagenesis induced via DNA polymerase eta. These mutations can occur in healthy lymphoid cells as part of a somatic hypermutation process, which introduces mutations to antibody-coding sequence in order to generate sequence variability and produce antibodies with higher specificity (Seki et al., 2005). SBS9 activity is elevated in Lymph-CLL samples that possess immunoglobulin gene hypermutation (Alexandrov et al., 2020, Gerstung et al., 2020), a mutational process that typically occurs early in tumor development (Dentro et al., 2021, Seifert et al., 2012).
Interestingly, changes in signature activity were distributed at many loci in Lymph-CLL samples, whereas in B-cell nonHodgkin lymphoma (Lymph-BNHL), which also undergoes polymerase eta dependent somatic hypermutation, changes in signature activity were concentrated on chromosome 14, close to the immunoglobulin gene hypermutation (IGH) locus. While the etiologies of SBS5 and SBS40 are unknown, their activity correlates with patient age, and SBS5 has been associated with proliferation (Alexandrov et al., 2020, Franco et al., 2019).

Lymph-CLL signature activity has previously been seen to be associated with evolutionary timing. Similar cancers also evolve in consistent patterns (Dentro et al., 2021, Rubanova et al., 2020, Campbell et al., 2020). In keeping with this, we observe recurrent changepoints that may be indicative of important evolutionary (and shared) changes that occur over the progression of these tumors, such as timing-dependent changes in chromatin state at these regions. Regional mutation rates and mutational signatures are influenced by chromatin state, and under normal cell growth conditions we would expect to see higher mutation rates in heterochromatic than euchromatic regions (Vöhringer et al., 2021, Yaacov et al., 2021). However, the compounding DNA damage and dysfunction in DNA repair that occurs as cancers develop could induce changes in chromatin state, making regions vulnerable to mutations late in development that were not vulnerable during early cancer formation. Ergo, if the chromatin state at a particular region changes during tumor evolution, this may also manifest as a regional change in signature activities.

\section{Changepoints are not associated with any sin-} gle phenomenon. We examined the correlation between changepoint locations and changes in gene density, mutation density, and when available, tissue-specific measures of chromatin accessibility (Polak et al., 2015) to investigate what underlying genomic or epigenomic features could account for the observed distribution of changepoints. Given that tissue-specific or cancer-specific DNAse-Seq or ATACSeq data is not available for all tumor types in our dataset, we cannot draw broad conclusions about the relationship between local changes in chromatin accessibility and changepoint placement. However, it is interesting to note that most melanoma samples display low correlation between DNAseI accessibility index and changepoint occurrence (mean correlation $=0.12$ ), thus it does not seem that local chromatin state changes alone can explain changes in signature activity (Fig. 5A-B). Changes in gene density or mutation density alone also fail to explain the distribution of observed changepoints. For instance, the mean correlations between changepoint occurrence and gene and mutation density across 74 melanoma samples are 0.13 and 0.11 , respectively (Fig. 5CD). Changepoints occur in both gene and mutation-dense and gene and mutation-poor regions, changes in both variables are often unaccompanied by changepoints, and we could observe no obvious biased placement of changepoint with respect to these variables. Furthermore, recurrent changepoints occur across multiple samples and cancer types, where these 

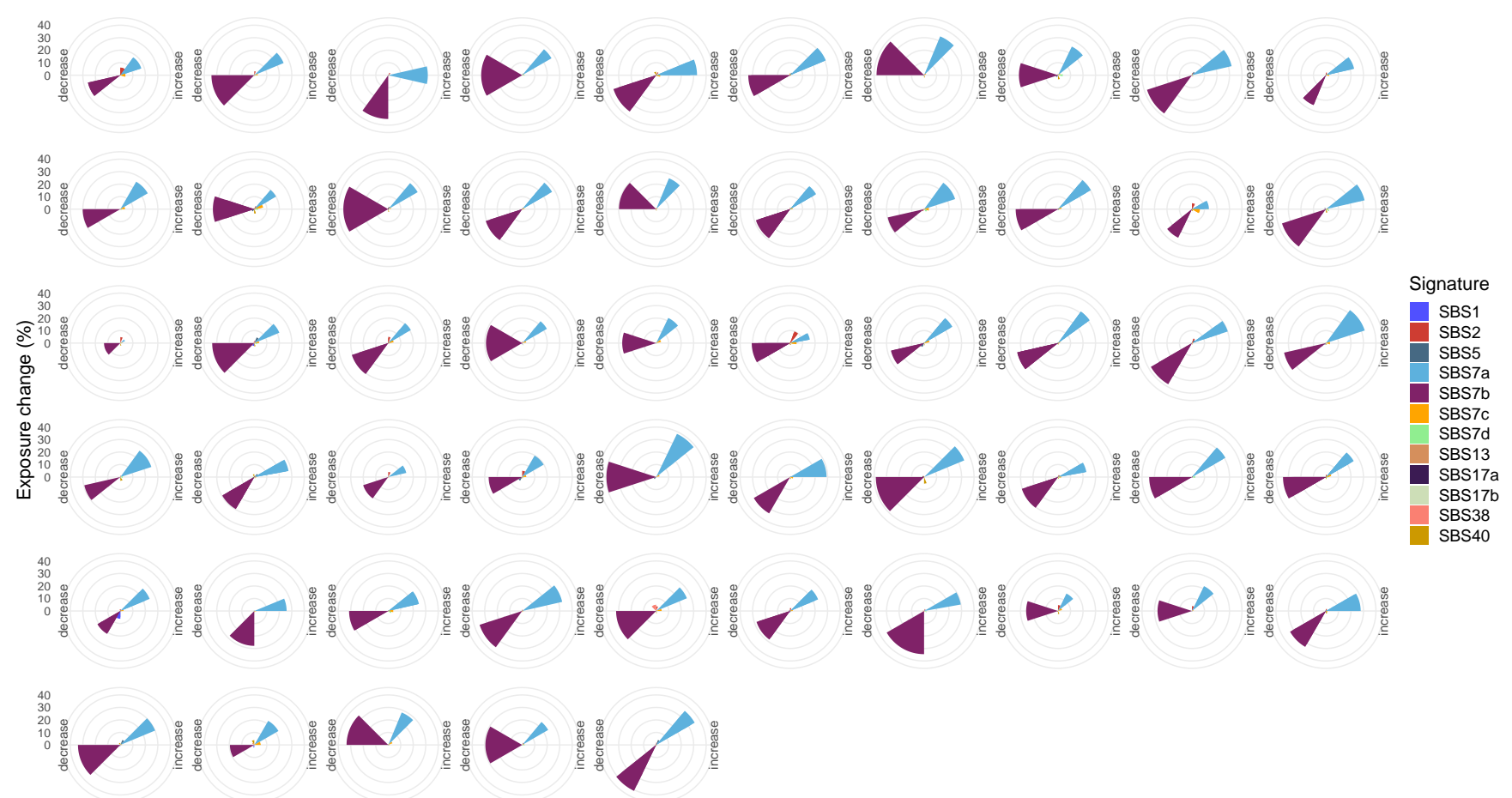

Fig. 3. Similar signature changes at the 1:47,000,000-1:51,000,000 region in 55/107 melanoma samples. Each polar coordinate chart shows the changes in mutational signature activity in a melanoma sample for a single recurrent changepoint region, at 1:47,000,000-1:51,000,000. Signatures on the left half of the chart decrease in activity at the changepoint and signatures on the right half increase in activity at the changepoint. The height of each slice denotes the numerical change in signature activity. The width of the slice indicates how many signatures increased or decreased at 1:47,000,000-1:51,000,000 in that sample (wider slice $=$ few signatures increasing, or vice versa).

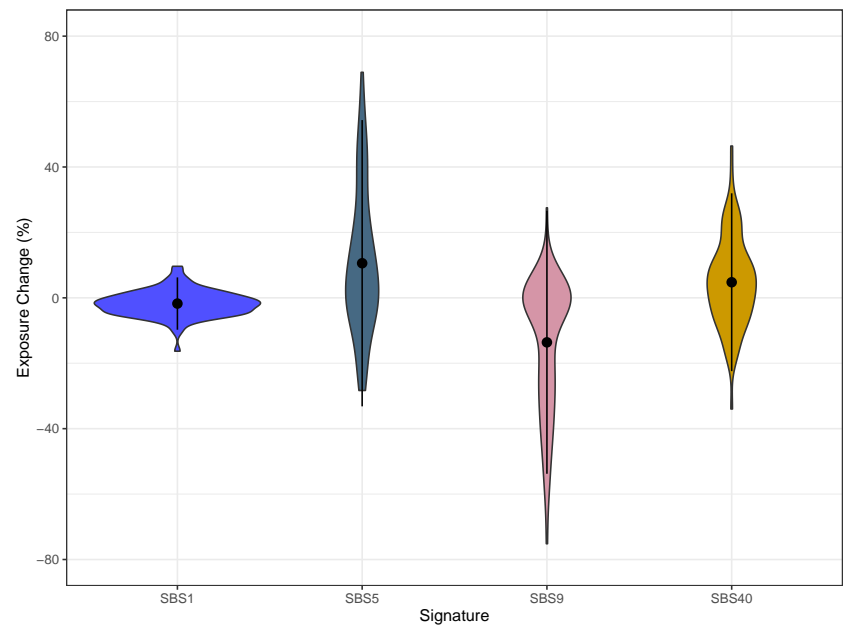

Fig. 4. Activity change in four SBS signatures during cancer evolution in 90 Lymph-CLLs. Violin plots showing the distribution of signature activity changes ( $y$ axis) from early to late stages of cancer development constructed using TrackSig (Rubanova et al., 2020, Harrigan et al., 2020, ?) for 90 chronic lymphocytic leukemia samples. Dot indicates means, vertical line spans $+/$ - one standard deviation.

\section{features vary.}

To further investigate if changes in mutation density affect changepoint placement, we also analyzed the distances between changepoints and copy number aberrations (CNAs), and the distances between changepoints and kataegis events. We conducted a randomization test to determine, for each sample, if a significant proportion of the changepoints in the sample overlap with a CNA. We found that overall, 60/426 samples with changepoints have a higher propor- tion of changepoints overlapping with a CNA than would be expected at random. Cervical cancers have the highest degree of changepoint/CNA co-occurrence, with 7 of 13 samples with changepoints displaying significant overlap. No Lymph-CLL, Prost-AdenoCA, Stomach-AdenoCA, or Colorect-AdenoCA samples showed significant changepoint/CNA overlap. These results emphasize that the changepoints detected by TrackSig reflect genuine shifts in mutational composition, not just mutation density. Further, we find that structural variation in the genome cannot explain the observed distribution of mutational signature activity changes.

Across the 2044/3059 changepoints within samples containing a kataegis event (Dentro et al., 2021, Nik-Zainal et al., 2012), these events were often distantly separated. The mean and median distances of changepoints to the nearest kataegis event were $174 \mathrm{Mb}$ and $10 \mathrm{Mb}$, respectively, although some are relatively close: 634/2044 changepoints were located within $1 \mathrm{Mb}$ from a kataegis event, suggesting that kataegis is one potential cause of a changepoint but not the majority cause. We observed a similar pattern when analyzing recurrent changepoints. The mean and median distances to a kataegis event among recurrent changepoints were $173 \mathrm{Mb}$ and $4 \mathrm{Mb}$ respectively, and 735/1159 changepoints were located $>1 \mathrm{Mb}$ away from their nearest kataegis event. One reason for the partial association of kataegis and changepoints may be that the vast majority of kataegis events involve APOBEC deaminases, which are represented by SBS2 and SBS13 (Alexandrov et al., 2020, Nik-Zainal et al., 2012). 
In this case, the hypermutation would result in a significant change in the local activity of these signatures, which would be detected by GenomeTrackSig. The localized hypermutation of the IGH locus in Lymph-BNHL may result in a SBS9enriched kataegic event, which may explain both the high incidence of kataegis in Lymph-BNHL as well as the proximity of kataegis to the recurrent changepoints in that cancer (mean and median distances $=7 \mathrm{Mb}$ and $6 \mathrm{Mb}$, respectively).

Lymph-BNHL exhibits at least one polymerase eta-driven hypermutation hotspot on every chromosome (Campbell et al., 2020), however changepoints were almost exclusively found on chromosome 14 (Fig. 2). In contrast, Lymph-CLL contains many recurrent change points across different chromosomes (Fig. 2) but relatively few kataegis foci (Campbell et al., 2020). Although these two cancers have commonalities in their activities of SBS1, SBS9, SBS5, and SBS40 (Alexandrov et al., 2020) as well as being the only two cancer types known to display polymerase eta-driven kataegis (Campbell et al., 2020), within them we observe nearly opposite relationships between GenomeTrackSig changepoints and kataegis foci. In summary, kataegis has some association with GenomeTrackSig changepoints but it does not explain the majority of changepoints and its presence does not always give rise to a changepoint.

\section{Discussion}

Here we have introduced a new method, GenomeTrackSig, to detect region-specific changes in mutational signature activity within cancer genomes. Using this method we have demonstrated frequent changes in mutational signature activities over large chromosomal domains in a variety of cancers. We have also found a surprising number of recurrent changepoints in signature activities shared across cancers of the same type and among cancers of different types.

The scale, $>1 \mathrm{Mb}$, at which GenomeTrackSig can generally detect changes in mutational signature activity is much larger than regional factors already known to influence mutation rates, which makes it surprising that we see so many changepoints. For example, regional mutation rate varies due to changes in replication timing, differential activity of DNA repair mechanisms, and chromatin accessibility (Schuster-Böckler and Lehner, 2012, Supek and Lehner, 2019, Vöhringer et al., 2021, Yaacov et al., 2021, Supek and Lehner, 2015, Zheng et al., 2014). In samples with many mutations, in which a changepoint can demarcate smaller regions ( $<1 \mathrm{Mb}$ in size), we may be able to attribute changes in signature activities to some of these factors that cause regional differences in the somatic mutation rate, especially if these factors change substantially over tumor development (see below). However, our effort to quantify this phenomenon using readily-measured genomic features - gene density, mutation density, structural and copy number variants, or kataegis (Fig. 5) - established that the signature changes we are detecting reflect a genuine shift in underlying mutational distributions and cannot be fully explained by these local, smaller-scale factors alone (Fig. 5).

Perhaps, instead our changepoints demarcate large-scale chromosomal organization, with each large domain having its own underlying mutational distribution driven by domainspecific DNA damage and repair dynamics. Given that signature changes at recurrent changepoints are often consistent within a tissue yet variable across tissues, it is possible that such chromosomal domains can occur at similar locations in multiple tissue types yet exert tissue-specific effects on the mutational landscape. One intriguing possibility is that these large-scale domains are delineated by the 3D organization of the cancer genome - representing, for example, larger topologically associating domains (TADs) Yu and Ren (2017).

The signature dynamics at the most common changepoint in melanoma samples support our hypothesis that changepoints are driven by chromatin state variation over large scales. The changepoint region at 1:47,000,000-51,000,000 is found in 77 samples from 5 tumor types, 55 of those melanomas. As shown in Fig. 4, all 55 melanomas display strikingly consistent decreases in SBS7b activity and increases in SBS7a activity at this changepoint. An analysis of the genomic properties influencing mutational signature activities by Vöhringer and colleagues (2021), using a de novo signature extraction method, identified two signatures exclusively occurring in skin cancers which highly resemble SBS7a and SBS7b in terms of their mutational distributions and associations with UV exposure. Their study found that the SBS7a-like signature has high activity in quiescent chromatin, whereas SBS7b-like is enriched in active chromatin and has a strong transcriptional strand bias. They suggested this differential signature activity across chromatin states reflects different operative DNA repair mechanisms. In this scenario, SBS7a-like activity reflects UV damage cleared by global genome nucleotide excision repair (GGNER), which operates in quiescent and active regions, and SBS7b-like activity reflects damage cleared by a combination of GG-NER and transcription-coupled nucleotide excision repair (TC-NER), the latter of which operates in open chromatin and is activated by template strand lesions on actively transcribed genes (Vöhringer et al., 2021). Therefore, the activity shifts between SBS7a and SBS7b that we observe at recurrent changepoint regions in melanomas may reflect large-scale changes in chromatin state, coupled with changes in active DNA repair processes.

Based on signature activity profiles from Lymph-CLL samples, we also hypothesize that large-scale changes in chromatin state sometimes occur in a timing-dependent manner. We observe changepoints in which early-development signatures decrease in activity and late-development signatures increase in activity, and vice versa. Cancer development is marked by a redistribution of the mutational landscape, as exposures to mutagens and DNA repair failures strip active genes in euchromatic regions of the preferential repair that they normally receive (Supek and Lehner 2019). Thus, we may see higher activity of late-development signatures in regions that normally have a low mutation rate, but which become vulnerable to mutation during subclonal expansion when different mutational processes are active than were early on. Conversely, late-replicating regions of closed 
bioRxiv preprint doi: https://doi.org/10.1101/2022.01.23.477261; this version posted February 27, 2022. The copyright holder for this preprint (which was not certified by peer review) is the author/funder, who has granted bioRxiv a license to display the preprint in perpetuity. It is made available under aCC-BY-NC 4.0 International license.

D Changepoints are not associated with any single phenomenon

A

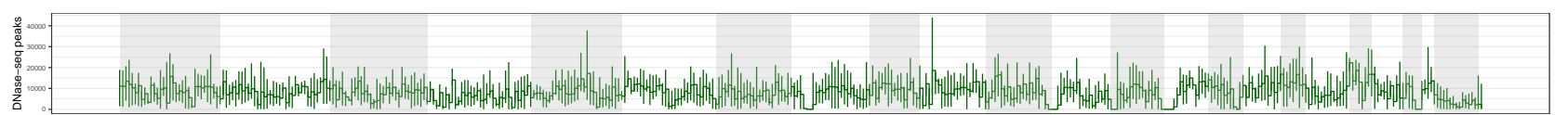
(1)
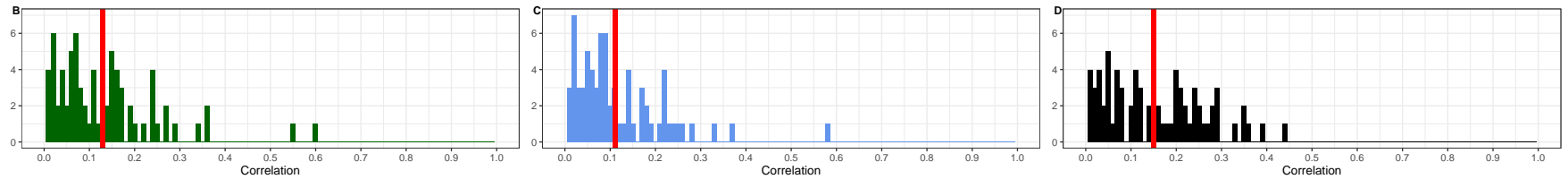

Fig. 5. The association of signature activity profiles and chromatin accessibility, gene density, and mutation density profiles. A: Signature activity profile (chromosome-wise) for a representative melanoma sample with 94,000 mutations and four changepoints. Colored lines denote signature activities across bins of 200 mutations. Alternating gray and white bars denote chromosomal boundaries, and vertical blue lines show centromere positions. Red vertical lines show changepoint locations, and the opacity of these lines denotes confidence in that changepoint's location. Above, black line plot depicts mutation density at each bin across the genome. Mutation density is normalized such that the bin with the highest density throughout the genome is scaled to one and the bin with the minimum density is scaled to zero. Above, blue area plot represents the average gene density at each bin, as determined from gene counts in hg19. Gene density is normalized in the same manner as mutation density. Above, green line plot depicts chromatin accessibility throughout a primary melanocyte genome as determined via DNAse-I accessibility index (Polak et al., 2015). Horizontal lines at each bin show the average chromatin accessibility across each bin, and vertical lines depict the range of chromatin accessibility values within each bin. B, C, and D show the distribution of correlation values between changepoint locations and DNAse-I accessibility index, gene density, and mutation density, respectively, across the 74 melanoma samples which contain changepoints. Mean correlation is highlighted in red on each plot.

chromatin might display higher activity of early-development signatures since these tend to have higher mutation rates under normal conditions.

Via mutational signature analysis, we have identified that mutational signature activities change over chromosomal domains. These changes can be highly consistent on multiple levels. We hypothesize that, among other factors, our method is detecting the impacts of large-scale changes in chromatin state. Recurrent changepoints in melanoma samples provide compelling evidence that regions on either side of a changepoint differ in terms of their chromatin accessibility and DNA repair dynamics. Furthermore, changepoints that appear in multiple tissue types raise the possibility that such wide-scale epigenomic changes are common events in the course of cancer development. These results call for further exploration into how factors like chromatin state and DNA repair mechanisms vary over wider domains, and which elements of these changes are characteristic of many cancers and which are tissue-specific. Interestingly, changepoints sometimes reflect a shift in activity between signatures characteristic of early tumor development and signatures characteristic of subclonal expansion. Thus, we hypothesize that large-scale changes in chromatin accessibility may also occur in a timing-dependent manner, and can demarcate regions that are more likely to be impacted by mutations early or late in a cancer's evolutionary trajectory. This finding may be clinically relevant given that intra-tumor heterogeneity is a mechanism of therapeu- tic resistance and therefore presents significant challenges for treatment (Maley et al., 2006, McGranahan and Swanton, 2017, Mroz et al., 2013). Therefore, genome-wide mutational signature analysis can help us further characterize and localize the genomic and epigenomic changes that occur during tumor development.

\section{ACKNOWLEDGEMENTS}

$\mathrm{QM}$ is a CCAI chair and is partially supported by a $\mathrm{NIH} / \mathrm{NCl}$ Cancer Center Support Grant P30 CA008748

\section{References}

Ludmil B. Alexandrov, Jaegil Kim, Nicholas J. Haradhvala, Mi Ni Huang, Alvin Wei Tian Ng, Yang Wu, Arnoud Boot, Kyle R. Covington, Dmitry A. Gordenin, Erik N. Bergstrom, S. M. Ashiqul Islam, Nuria Lopez-Bigas, Leszek J. Klimczak, John R. McPherson, Sandro Morganella, Radhakrishnan Sabarinathan, David A. Wheeler, Ville Mustonen, PCAWG Mutational Signatures Working Group, Gad Getz, Steven G. Rozen, Michael R. Stratton, and PCAWG Consortium. The repertoire of mutational signatures in human cancer. Nature, 578(7793):94-101, February 2020. ISSN 1476-4687. doi: 10.1038/s41586-020-1943-3.

Stefan C. Dentro, Ignaty Leshchiner, Kerstin Haase, Maxime Tarabichi, Jeff Wintersinger, Amit G. Deshwar, Kaixian Yu, Yulia Rubanova, Geoff Macintyre, Jonas Demeulemeester, Ignacio Vázquez-García, Kortine Kleinheinz, Dimitri G. Livitz, Salem Malikic, Nilgun Donmez, Subhajit Sengupta, Pavana Anur, Clemency Jolly, Marek Cmero, Daniel Rosebrock, Steven E. Schumacher, Yu Fan, Matthew Fittall, Ruben M. Drews, Xiaotong Yao, Thomas B. K. Watkins, Juhee Lee, Matthias Schlesner, Hongtu Zhu, David J. Adams, Nicholas McGranahan, Charles Swanton, Gad Getz, Paul C. Boutros, Marcin Imielinski, Rameen Beroukhim, S. Cenk Sahinalp, Yuan Ji, Martin Peifer, Inigo Martincorena, Florian Markowetz, Ville Mustonen, Ke Yuan, Moritz Gerstung, Paul T. Spellman, Wenyi Wang, Quaid D. Morris, David C. Wedge, Peter Van Loo, and PCAWG Evolution and Heterogeneity Working Group and the PCAWG Consortium. Characterizing genetic intra-tumor heterogeneity across 2,658 human cancer genomes. Cell, 184(8):2239-2254.e39, April 2021. ISSN 1097-4172. doi: 10.1016/j.cell.2021.03.009.

Moritz Gerstung, Clemency Jolly, Ignaty Leshchiner, Stefan C. Dentro, Santiago Gonzalez, Daniel Rosebrock, Thomas J. Mitchell, Yulia Rubanova, Pavana Anur, Kaixian Yu, Maxime Tarabichi, Amit Deshwar, Jeff Wintersinger, Kortine Kleinheinz, Ignacio Vázquez-García, Kerstin Haase, Lara Jerman, Subhajit Sengupta, Geoff Macintyre, Salem Malikic, Nilgun Donmez, 
bioRxiv preprint doi: https://doi.org/10.1101/2022.01.23.477261; this version posted February 27, 2022. The copyright holder for this preprint (which was not certified by peer review) is the author/funder, who has granted bioRxiv a license to display the preprint in perpetuity. It is made available under aCC-BY-NC 4.0 International license.

Dimitri G. Livitz, Marek Cmero, Jonas Demeulemeester, Steven Schumacher, Yu Fan, Xiaotong Yao, Juhee Lee, Matthias Schlesner, Paul C. Boutros, David D. Bowtell, Hongtu Zhu, Gad Getz, Marcin Imielinski, Rameen Beroukhim, S. Cenk Sahinalp, Yuan Ji, Martin Peifer, Florian Markowetz, Ville Mustonen, Ke Yuan, Wenyi Wang, Quaid D. Morris, Paul T. Spellman, David C. Wedge, and Peter Van Loo. The evolutionary history of 2,658 cancers. Nature, 578 (7793):122-128, February 2020. ISSN 1476-4687. doi: 10.1038/s41586-019-1907-7.

Yulia Rubanova, Ruian Shi, Caitlin F. Harrigan, Roujia Li, Jeff Wintersinger, Nil Sahin, Amit Deshwar, PCAWG Evolution and Heterogeneity Working Group, Quaid Morris, and PCAWG Consortium. Reconstructing evolutionary trajectories of mutation signature activities in cancer using TrackSig. Nature Communications, 11(1):731, February 2020. ISSN 2041-1723. doi: 10.1038/s41467-020-14352-7.

Caitlin F. Harrigan, Yulia Rubanova, Quaid Morris, and Alina Selega. TrackSigFreq: subclona reconstructions based on mutation signatures and allele frequencies. Pacific Symposium on Biocomputing. Pacific Symposium on Biocomputing, 25:238-249, 2020. ISSN 2335-6936.

Abel Gonzalez-Perez, Radhakrishnan Sabarinathan, and Nuria Lopez-Bigas. Local Determinants of the Mutational Landscape of the Human Genome. Cell, 177(1):101-114, March 2019. ISSN 1097-4172. doi: 10.1016/j.cell.2019.02.051.

Nicholas J. Haradhvala, Paz Polak, Petar Stojanov, Kyle R. Covington, Eve Shinbrot, Julian Hess, Esther Rhienbey, Jaegil Kim, Yosef Maruvka, Lior Z. Braunstein, Atanas Kamburov, Philip C. Hanawalt, David A. Wheeler, Amnon Koren, Michael S. Lawrence, and Gad Getz. Mutational strand asymmetries in cancer genomes reveal mechanisms of dna damage and repair. Cell, 164(3):538-549, January 2016. ISSN 0092-8674. doi: 10.1016/j.cell.2015.12.050.

Alan Hodgkinson, Ying Chen, and Adam Eyre-Walker. The large-scale distribution of somatic mutations in cancer genomes. Human Mutation, 33(1):136-143, January 2012. ISSN 10981004. doi: 10.1002/humu.21616.

Michael S. Lawrence, Petar Stojanov, Paz Polak, Gregory V. Kryokov, Kristian Cibulskis, Andrey Sivachenko, Scott L. Carter, Chip Stewart, Craig H. Mermel, Steven A. Roberts, Adam Kiezun, Peter S. Hammerman, Aaron McKenna, Yotam Drier, Lihua Zou, Alex H. Ramos, Trevor J. Pugh, Nicolas Stransky, Elena Helman, Jaegil Kim, Carrie Sougnez, Lauren Ambrogio, Elizabeth Nickerson, Erica Shefler, Maria L. Cortés, Daniel Auclair, Gordon Saksena, Douglas Voet, Michael Noble, Daniel DiCara, Pei Lin, Lee Lichtenstein, David I. Heiman, Timothy Fennell, Marcin Imielinski, Bryan Hernandez, Eran Hodis, Sylvan Baca, Austin M. Dulak, Jens Lohr, Dan-Avi Landau, Catherine J. Wu, Jorge Melendez-Zajgla, Alfredo Hidalgo-Miranda, Amnon Koren, Steven A. McCarroll, Jaume Mora, Ryan S. Lee, Brian Crompton, Robert Onofrio, Melissa Parkin, Wendy Winckler, Kristin Ardlie, Stacey B. Gabriel, Charles W.M. Roberts, Jaclyn A. Biegel, Kimberly Stegmaier, Adam J. Bass, Levi A. Garraway, Matthew Meyerson, Todd R. Golub, Dmitry A. Gordenin, Shamil Sunyaev, Eric S. Lander, and Gad Getz. Mutational heterogeneity in cancer and the search for new cancer-associated genes. Nature, 499(7457):214-218, July 2013. ISSN 1476-4687. doi: 10.1038/nature12213.

Paz Polak, Michael S. Lawrence, Eric Haugen, Nina Stolzetski, Petar Stojanov, Robert E. Thurman, Levi A. Garraway, Sergei Mirkin, Gad Getz, John A. Stamatoyannopoulos, and Shamil R. Sunyaev. Reduced local mutation density in regulatory dna of cancer genomes is linked to dna repair. Nature Biotechnology, 32(1):71-75, January 2014. ISSN 1087-0156. doi: 10.1038/nbt.2778.

Paz Polak, Rosa Karlić, Amnon Koren, Robert Thurman, Richard Sandstrom, Michael S. Lawrence, Alex Reynolds, Eric Rynes, Kristian Vlahoviček, John A. Stamatoyannopoulos, and Shamil R. Sunyaev. Cell-of-origin chromatin organization shapes the mutational landscape of cancer. Nature, 518(7539):360-364, February 2015. ISSN 1476-4687. doi: 10.1038/nature 14221

Benjamin Schuster-Böckler and Ben Lehner. Chromatin organization is a major influence on regional mutation rates in human cancer cells. Nature, 488(7412):504-507, August 2012. ISSN 1476-4687. doi: 10.1038/nature11273.

Vladimir B. Seplyarskiy and Shamil Sunyaev. The origin of human mutation in light of genomic data. Nature Reviews. Genetics, 22(10):672-686, October 2021. ISSN 1471-0064. doi: 10.1038/s41576-021-00376-2.

Fran Supek and Ben Lehner. Scales and mechanisms of somatic mutation rate variation across the human genome. DNA repair, 81:102647, September 2019. ISSN 1568-7856. doi: 10. 1016/j.dnarep.2019.102647.

Harald Vöhringer, Arne Van Hoeck, Edwin Cuppen, and Moritz Gerstung. Learning mutational signatures and their multidimensional genomic properties with TensorSignatures. Nature Com munications, 12(1):3628, June 2021. ISSN 2041-1723. doi: 10.1038/s41467-021-23551-9.

Adar Yaacov, Oriya Vardi, Britny Blumenfeld, Avraham Greenberg, Dashiell J. Massey, Amnon Koren, Sheera Adar, Itamar Simon, and Shai Rosenberg. Cancer Mutational Processes Vary in Their Association with Replication Timing and Chromatin Accessibility. Cancer Research, 81 (24):6106-6116, December 2021. ISSN 1538-7445. doi: 10.1158/0008-5472.CAN-21-2039.

Fran Supek and Ben Lehner. Differential DNA mismatch repair underlies mutation rate variation across the human genome. Nature, 521(7550):81-84, May 2015. ISSN 1476-4687. doi: 10.1038 /nature 14173 .

Christina L. Zheng, Nicholas J. Wang, Jongsuk Chung, Homayoun Moslehi, J. Zachary Sanborn, Joseph S. Hur, Eric A. Collisson, Swapna S. Vemula, Agne Naujokas, Kami E. Chiotti, Jeffrey B. Cheng, Hiva Fassihi, Andrew J. Blumberg, Celeste V. Bailey, Gary M. Fudem, Frederick G. Mihm, Bari B. Cunningham, Isaac M. Neuhaus, Wilson Liao, Dennis H. Oh, James E. Cleaver, Philip E. LeBoit, Joseph F. Costello, Alan R. Lehmann, Joe W. Gray, Paul T. Spellman, Sarah T. Arron, Nam Huh, Elizabeth Purdom, and Raymond J. Cho. Transcription Restores DNA Repair to Heterochromatin, Determining Regional Mutation Rates in Cancer Genomes. Cell Reports, 9(4):1228-1234, November 2014. ISSN 2211-1247. doi: 10.1016/j.celrep.2014.10.031

Peter J. Campbell, Gad Getz, Jan O. Korbel, Joshua M. Stuart, Jennifer L. Jennings, Lincoln D. Stein, Marc D. Perry, Hardeep K. Nahal-Bose, B. F. Francis Ouellette, Constance H. Li, Esther Rheinbay, G. Petur Nielsen, Dennis C. Sgroi, Chin-Lee Wu, William C. Faquin, Vikram Deshpande, Paul C. Boutros, Alexander J. Lazar, Katherine A. Hoadley, David N. Louis, L. Jonathan Dursi, Christina K. Yung, Matthew H. Bailey, Gordon Saksena, Keiran M. Raine, Ivo Buchhalter, Kortine Kleinheinz, Matthias Schlesner, Junjun Zhang, Wenyi Wang, David A. Wheeler, Li Ding, Jared T. Simpson, Brian D. O'Connor, Sergei Yakneen, Kyle Ellrott, Naoki Miyoshi, Adam P. Butler, Romina Royo, Solomon I. Shorser, Miguel Vazquez, Tobias Rausch, Grace Tiao, Sebastian M. Waszak, Bernardo Rodriguez-Martin, Suyash
Shringarpure, Dai-Ying Wu, German M. Demidov, Olivier Delaneau, Shuto Hayashi, Seiya Imoto, Nina Habermann, Ayellet V. Segre, Erik Garrison, Andy Cafferkey, Eva G. Alvarez, José María Heredia-Genestar, Francesc Muyas, Oliver Drechsel, Alicia L. Bruzos, Javier Temes, Jorge Zamora, Adrian Baez-Ortega, Hyung-Lae Kim, R. Jay Mashl, Kai Ye, Anthony DiBiase, Kuan-lin Huang, Ivica Letunic, Michael D. McLellan, Steven J. Newhouse, Tal Shmaya, Sushant Kumar, David C. Wedge, Mark H. Wright, Venkata D. Yellapantula, Mark Gerstein, Ekta Khurana, Tomas Marques-Bonet, Arcadi Navarro, Carlos D. Bustamante, Reiner Siebert, Hidewaki Nakagawa, Douglas F. Easton, Stephan Ossowski, Jose M. C. Tubio, Francisco M. De La Vega, Xavier Estivill, Denis Yuen, George L. Mihaiescu, Larsson Omberg, Vincent Ferretti, Radhakrishnan Sabarinathan, Oriol Pich, Abel GonzalezPerez, Amaro Taylor-Weiner, Matthew W. Fittall, Jonas Demeulemeester, Maxime Tarabichi, Nicola D. Roberts, Peter Van Loo, Isidro Cortés-Ciriano, Lara Urban, Peter Park, Bin Zhu, Esa Pitkänen, Yilong Li, Natalie Saini, Leszek J. Klimczak, Joachim Weischenfeldt, Nikos Sidiropoulos, Ludmil B. Alexandrov, Raquel Rabionet, Georgia Escaramis, Mattia Bosio, Aliaksei Z. Holik, Hana Susak, Aparna Prasad, Serap Erkek, Claudia Calabrese, Benjamin Raeder, Eoghan Harrington, Simon Mayes, Daniel Turner, Sissel Juul, Steven A. Roberts, Lei Song, Roelof Koster, Lisa Mirabello, Xing Hua, Tomas J. Tanskanen, Marta Tojo, Jieming Chen, Lauri A. Aaltonen, Gunnar Rätsch, Roland F. Schwarz, Atul J. Butte, Alvis Brazma, Stephen J. Chanock, Nilanjan Chatterjee, Oliver Stegle, Olivier Harismendy, G. Steven Bova, Dmitry A. Gordenin, David Haan, Lina Sieverling, Lars Feuerbach, Don Chalmers, Yann Joly, Bartha Knoppers, Fruzsina Molnár-Gábor, Mark Phillips, Adrian Thorogood, David Townend, Mary Goldman, Nuno A. Fonseca, Qian Xiang, Brian Craft, Elena Piñeiro-Yáñez, Alfonso Muñoz, Robert Petryszak, Anja Füllgrabe, Fatima Al-Shahrour, Maria Keays, David Haussler, John Weinstein, Wolfgang Huber, Alfonso Valencia, Irene Papatheodorou, Jingchun Zhu, Yu Fan, David Torrents, Matthias Bieg, Ken Chen, Zechen Chong, Kristian Cibulskis, Roland Eils, Robert S. Fulton, Josep L. Gelpi, Santiago Gonzalez, Ivo G. Gut, Faraz Hach, Michael Heinold, Taobo Hu, Vincent Huang, Barbara Hutter, Natalie Jäger, Jongsun Jung, Yogesh Kumar, Christopher Lalansingh, Ignaty Leshchiner, Dimitri Livitz, Eric Z. Ma, Yosef E. Maruvka, Ana Milovanovic, Morten Muhlig Nielsen, Nagarajan Paramasivam, Jakob Skou Pedersen, Montserrat Puiggròs, S. Cenk Sahinalp, Iman Sarrafi, Chip Stewart, Miranda D. Stobbe, Jeremiah A. Wala, Jiayin Wang, Michael Wendl, Johannes Werner, Zhenggang Wu, Hong Xue, Takafumi N. Yamaguchi, Venkata Yellapantula, Brandi N. Davis-Dusenbery, Robert L. Grossman, Youngwook Kim, Michael C. Heinold, Jonathan Hinton, David R. Jones, Andrew Menzies, Lucy Stebbings, Julian M. Hess, Mara Rosenberg, Andrew J. Dunford, Manaswi Gupta, Marcin Imielinski, Matthew Meyerson, Rameen Beroukhim, Jüri Reimand, Priyanka Dhingra, Francesco Favero, Stefan Dentro, Jeff Wintersinger, Vasilisa Rudneva, Ji Wan Park, Eun Pyo Hong, Seong Gu Heo, André Kahles, Kjong-Van Lehmann, Cameron M. Soulette, Yuichi Shiraishi, Fenglin Liu, Yao He, Deniz Demircioğlu, Natalie R. Davidson, Liliana Greger, Siliang Li, Dongbing Liu, Stefan G. Stark, Fan Zhang, Samirkumar B. Amin, Peter Bailey, Aurélien Chateigner, Milana Frenkel-Morgenstern, Yong Hou, Matthew R. Huska, Helena Kilpinen, Fabien C. Lamaze, Chang Li, Xiaobo Li, Xinyue Li, Xingmin Liu, Maximillian G. Marin, Julia Markowski, Tannistha Nandi, Akinyemi I. Ojesina, Qiang Pan-Hammarström, Peter J. Park, Chandra Sekhar Pedamallu, Hong Su, Patrick Tan, Bin Tean Teh, Jian Wang, Heng Xiong, Chen Ye, Christina Yung, Xiuqing Zhang, Liangtao Zheng, Shida Zhu, Philip Awadalla, Chad J. Creighton, Kui Wu, Huanming Yang, Jonathan Göke, Zemin Zhang, Angela N. Brooks, Matthew W. Fittall, Iñigo Martincorena, Carlota Rubio-Perez, Malene Juul, Steven Schumacher, Ofer Shapira, David Tamborero, Loris Mularoni, Henrik Hornshøj, Jordi Deu-Pons, Ferran Muiños, Johanna Bertl, Qianyun Guo, Abel Gonzalez-Perez, Qian Xiang, and The ICGC/TCGA Pan-Cancer Analysis of Whole Genomes Consortium. Pan-cancer analysis of whole genomes. Nature, 578(7793):82-93, February 2020. ISSN 1476-4687. doi: 10.1038/s41586-020-1969-6.

R. Killick, P. Fearnhead, and I. A. Eckley. Optimal Detection of Changepoints With a Linea Computational Cost. Journal of the American Statistical Association, 107(500):1590-1598, December 2012. ISSN 0162-1459. doi: 10.1080/01621459.2012.737745.

T.K. Moon. The expectation-maximization algorithm. IEEE Signal Processing Magazine, 13(6): 47-60, November 1996. ISSN 1558-0792. doi: 10.1109/79.543975. Conference Name: IEEE Signal Processing Magazine.

John G. Tate, Sally Bamford, Harry C. Jubb, Zbyslaw Sondka, David M. Beare, Nidhi Bindal, Harry Boutselakis, Charlotte G. Cole, Celestino Creatore, Elisabeth Dawson, Peter Fish, Bhavana Harsha, Charlie Hathaway, Steve C. Jupe, Chai Yin Kok, Kate Noble, Laura Ponting, Christopher C. Ramshaw, Claire E. Rye, Helen E. Speedy, Ray Stefancsik, Sam L. Thompson, Shicai Wang, Sari Ward, Peter J. Campbell, and Simon A. Forbes. COSMIC: the Catalogue Of Somatic Mutations In Cancer. Nucleic Acids Research, 47(D1):D941-D947, January 2019. ISSN 1362-4962. doi: 10.1093/nar/gky1015.

Hira Lal Goel, Cheng Chang, Bryan Pursell, Irwin Leav, Stephen Lyle, Hualin Simon Xi, Chung-Cheng Hsieh, Helty Adisetiyo, Pradip Roy-Burman, Ilsa M. Coleman, Peter S. Nelson, Robert L. Vessella, Roger J. Davis, Stephen R. Plymate, and Arthur M. Mercurio. VEGF/neuropilin-2 regulation of Bmi-1 and consequent repression of IGF-IR define a novel mechanism of aggressive prostate cancer. Cancer Discovery, 2(10):906-921, October 2012. ISSN 2159-8290. doi: 10.1158/2159-8290.CD-12-0085.

Mariya S. Liyasova, Ke Ma, and Stanley Lipkowitz. Molecular pathways: cbl proteins in tumorigenesis and antitumor immunity-opportunities for cancer treatment. Clinical Cancer Research: An Official Journal of the American Association for Cancer Research, 21(8):1789-1794, April 2015. ISSN 1557-3265. doi: 10.1158/1078-0432.CCR-13-2490.

Kou-Juey Wu. The role of miRNA biogenesis and DDX17 in tumorigenesis and cancer stemness. Biomedical Journal, 43(2):107-114, April 2020. ISSN 2319-4170. doi: 10.1016/j.bj.2020.03. 001 .

Mineaki Seki, Patricia J Gearhart, and Richard D Wood. DNA polymerases and somatic hypermutation of immunoglobulin genes. EMBO Reports, 6(12):1143-1148, December 2005. ISSN 1469-221X. doi: 10.1038/sj.embor.7400582.

Marc Seifert, Ludger Sellmann, Johannes Bloehdorn, Frederik Wein, Stephan Stilgenbauer, Jan Dürig, and Ralf Küppers. Cellular origin and pathophysiology of chronic lymphocytic leukemia. The Journal of Experimental Medicine, 209(12):2183-2198, November 2012. ISSN 15409538. doi: 10.1084/jem.20120833.

Irene Franco, Hafdis T. Helgadottir, Aldo Moggio, Malin Larsson, Peter Vrtačnik, Anna Johansson, Nina Norgren, Pär Lundin, David Mas-Ponte, Johan Nordström, Torbjörn Lundgren, 
bioRxiv preprint doi: https://doi.org/10.1101/2022.01.23.477261; this version posted February 27, 2022. The copyright holder for this preprint (which was not certified by peer review) is the author/funder, who has granted bioRxiv a license to display the preprint in perpetuity. It is made available under aCC-BY-NC 4.0 International license.

D Changepoints are not associated with any single phenomenon

Peter Stenvinkel, Lars Wennberg, Fran Supek, and Maria Eriksson. Whole genome DNA sequencing provides an atlas of somatic mutagenesis in healthy human cells and identifies a tumor-prone cell type. Genome Biology, 20:285, December 2019. ISSN 1474-7596. doi: 10.1186/s13059-019-1892-z.

Serena Nik-Zainal, Ludmil B. Alexandrov, David C. Wedge, Peter Van Loo, Christopher D. Greenman, Keiran Raine, David Jones, Jonathan Hinton, John Marshall, Lucy A. Stebbings, Andrew Menzies, Sancha Martin, Kenric Leung, Lina Chen, Catherine Leroy, Manasa Ramakrishna, Richard Rance, King Wai Lau, Laura J. Mudie, Ignacio Varela, David J. McBride, Graham R. Bignell, Susanna L. Cooke, Adam Shlien, John Gamble, Ian Whitmore, Mark Maddison, Patrick S. Tarpey, Helen R. Davies, Elli Papaemmanuil, Philip J. Stephens, Stuart McLaren, Adam P. Butler, Jon W. Teague, Göran Jönsson, Judy E. Garber, Daniel Silver, Penelope Miron, Aquila Fatima, Sandrine Boyault, Anita Langerød, Andrew Tutt, John W.M. Martens, Samuel A.J.R. Aparicio, Åke Borg, Anne Vincent Salomon, Gilles Thomas, Anne-Lise Børresen-Dale, Andrea L. Richardson, Michael S. Neuberger, P. Andrew Futreal, Peter J. Campbell, and Michael R. Stratton. Mutational Processes Molding the Genomes of 21 Breast Cancers. Cell, 149(5-10):979-993, May 2012. ISSN 0092-8674. doi: 10.1016/j.cell.2012.04.024

Miao Yu and Bing Ren. The Three-Dimensional Organization of Mammalian Genomes. Annual Review of Cell and Developmental Biology, 33:265-289, October 2017. ISSN 1530-8995. doi: 10.1146/annurev-cellbio-100616-060531.

Carlo C. Maley, Patricia C. Galipeau, Jennifer C. Finley, V. Jon Wongsurawat, Xiaohong Li, Carissa A. Sanchez, Thomas G. Paulson, Patricia L. Blount, Rosa-Ana Risques, Peter S. Rabinovitch, and Brian J. Reid. Genetic clonal diversity predicts progression to esophageal adenocarcinoma. Nature Genetics, 38(4):468-473, April 2006. ISSN 1061-4036. doi: $10.1038 /$ ng 1768 .

Nicholas McGranahan and Charles Swanton. Clonal Heterogeneity and Tumor Evolution: Past, Present, and the Future. Cell, 168(4):613-628, February 2017. ISSN 1097-4172. doi: 10. 1016/j.cell.2017.01.018.

Edmund A. Mroz, Aaron D. Tward, Curtis R. Pickering, Jeffrey N. Myers, Robert L. Ferris, and James W. Rocco. High intratumor genetic heterogeneity is related to worse outcome in patients with head and neck squamous cell carcinoma. Cancer, 119(16):3034-3042, August 2013. ISSN 1097-0142. doi: 10.1002/cncr.28150. 